\title{
50 Jahre Fortschritte in der Arzneimitteltherapie - Rückblick und Ausblick
}

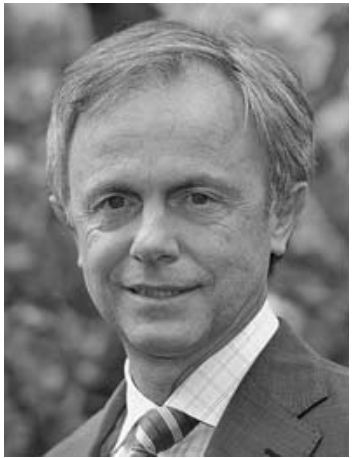

S. Endres
Früher war alles besser, sagen viele. Sicher mehr Lametta, aber wie sah es bei den Therapiemöglichkeiten von Krankheiten aus? Vor 50 Jahren standen zur Behandlung von Patienten mit Diabetes Typ 2 lediglich Insulin und der erste Sulfonylharnstoff, Tolbutamid, zur Verfügung. Metformin kam gerade in den Markt. Gegen Thrombosen gab es Vitamin-K-Antagonisten, Heparin und ASS, und gegen rheumatoide Arthritis und Multiple Sklerose nur die Allzweckwaffe Cortison. Bei bakteriellen Infektionen konnte man auf Streptomycin, Penicilline und Cephalosporine zurückgreifen, und Tumore wurden mit Chlorambucil, Cyclophosphamid sowie Methotrexat und zytostatischen Antibiotika behandelt.

Dieses aus heutiger Sicht doch sehr begrenzte therapeutische Arsenal und die auch daraus resultierende Lebenserwartung von lediglich 70 Jahren - 11 Jahre weniger als heutzutage - sind wahrlich kein Grund für Nostalgie.
Den Zielen der Paul-Martini-Stiftung entsprechend werden beim diesjährigen Jubiläums-Symposium führende Vertreter ihrer jeweiligen Fachdisziplin nachzeichnen, welche wesentlichen Entdeckungen und Erfindungen die Medizin über ihren Stand von 1966 hinaus gebracht haben. Und sie werden darstellen, woran nun gearbeitet wird, um die erreichten Therapiestandards noch zu überbieten.

Wesentlicher Bestandteil des Symposiums soll auch 2016 die Diskussion zwischen den Teilnehmern aus Wissenschaft, Industrie, Behörden und anderen Bereichen des Gesundheitswesens sein. Wir begrüßen Sie sehr herzlich zu dieser Jubiläumstagung.

\section{Stefan Endres}

Ludwig-Maximilians-Universität München 